\title{
Effects of Abdominal Control Feedback and Scapular Stabilization Exercise on Chronic Neck Pain
}

\author{
Sneha Ganu ${ }^{1}$, Ushma Gor ${ }^{2}$ \\ ${ }^{1}$ Associate Professor, MGM College of Physiotherapy, Navi Mumbai, Maharashtra, India. \\ ${ }^{2}$ Intern, MGM College of Physiotherapy, Navi Mumbai, Maharashtra, India.
}

Corresponding Author: Sneha Ganu

\begin{abstract}
Neck pain caused by functional impairment of the cervical spine is manageable with the appropriate intervention. This study aimed to prove that the addition of Abdominal Control Feedback to the conventional protocol, that included only Scapular Stabilization Exercises, would assist with pain minimization, increased available range of movement at the cervical spine, elevation in the endurance time of the deep flexor and extensor group of cervical muscles, better proprioception and an overall decrease in the disability caused due to the pain. A total of 40 participants with chronic mechanical neck pain in the age group of 18-40 years and a pain scale rating of less than 7 on the Visual Analog Scale were chosen and randomized into 2 groups. Group $1(n=20)$ received Scapular stabilization exercises without Abdominal control feedback for 6 weeks whereas Group $2(n=20)$ received Scapular stabilization exercises along with Abdominal control feedback for 6 weeks. Pain, range of motion, endurance, proprioception and disability were assessed before and after the interventions. There were significant intra group differences in pain, range, endurance, proprioception and disability in both the groups 1 and 2. There were significant inter group differences in pain and endurance siding with group 2. No significant inter group differences were observed in range of motion, proprioception and disability. This verified that the addition of abdominal control feedback to Scapular stabilization exercises aided in reducing neck pain and increasing endurance time in individuals with chronic mechanical neck pain.
\end{abstract}

Key Words: Neck pain, Abdominal control feedback, Scapular stabilization.

\section{INTRODUCTION}

Neck pain is becoming increasingly common throughout the world. The estimated one-year incidence of neck pain from available studies ranges between $10.4 \%$ and $21.3 \% .^{[1]}$ Mechanical neck pain refers to pain originating in the spine or its supporting structures, such as ligaments and muscles. ${ }^{[2]}$ Common examples of mechanical pain include pain arising from the facet joints (e.g. arthritis), discogenic pain, and myofascial pain. ${ }^{[3,4]}$ Much neck pain is not attributable to a specific disease or disorder and is labelled as 'soft-tissue' rheumatism or muscular / mechanical / postural neck pain. Mechanical stress to pain sensitive structures occurs in the absence of an inflammatory response. Thus, it is not a pathological problem but a mechanical one because signs of acute inflammation with constant pain are not present. ${ }^{[5,2]}$ If the mechanical stresses exceed the supporting capabilities of the tissue, breakdown ensues. Therefore, to prevent any further damage, early intervention and relief of mechanical stress is of key importance. ${ }^{[5]}$ The shoulder complex and scapula are closely linked to the cervical spine and often assessed along with it so as to isolate the pain causing 

pain.

structures and look for referred pain in the clinical setting. Neck pain has shown to affect muscle strength, deep neck flexor endurance, and muscle function capacity. ${ }^{[6]}$ The scapular stabilizers include static and muscular stabilizers. The static stabilizers include cohesive forces of the subscapular bursa, SC and AC joint ligaments and the scapulothoracic fascia. The muscular stabilizers include upper, middle, and lower trapezius, serratus anterior, levator scapulae and rhomboids. The serratus anterior and trapezius have their origins in the cervical region and thus affect the overall wellbeing in the neck region too. ${ }^{[7-10]}$ If the serratus anterior is held in a shortened position due to faulty postures like forward head and rounded shoulder, it leads to neck pain. ${ }^{[11-14]}$ Thus it is essential to improve serratus anterior activation to assist with better posture and reduce pain. Activation of the scapulothoracic muscles is considered a significant aspect of neck pain rehabilitation (Kibler et al., 2008; Kibler et al., 2010). Based on previous research, the conscious use of abdominal muscles during feedback exercises increases the involvement of Serratus anterior. ${ }^{[15]}$ There is an increased EMG activity of serratus anterior, upper, middle and lower trapezius during dynamic exercises while consciously contracting the abdominal muscles. ${ }^{[16]}$ Previous studies suggest muscle synergy between the oblique external (OE) muscle and serratus anterior(SA) during knee push up plus with ipsilateral leg extension (Maenhout et al., 2010; Kim et al., 2011). As a result of the leg extension and gluteus maximus activity, the thoracolumbar fascia is tightened and the lumbar spine extended. Compensatory ipsilateral external obliques muscle tension is created and transferred to the proximal tendon of the SA, leading to greater muscle fibre recruitment in the scapulothoracic muscle (Maenhout et al., 2010; Kim et al., 2011).

This study is oriented towards participants suffering from chronic mechanical neck pain. A protocol has been set in reference to other literature and is to be used as treatment for the participants of this study over an intervention period of 6 weeks. The goal of this study is to find the outcomes of abdominal control feedback addition to scapular stabilization exercise protocol on the pain, strength, proprioception, disability, endurance and range of motion in patients with chronic mechanical neck pain.

\section{Objectives:}

- To study effects on the cervical ROMs via goniometer.

- To study effects on muscle endurance using cervical flexor test and cervical extensor endurance test.

- To study effects on disability using the NDI.

- To study effects on pain using VAS.

- To study effects on proprioception using goniometer.

\section{MATERIALS AND METHODS Participants:}

40 Subjects having chronic mechanical neck pain in the age group of 18-40 years were chosen by convenient sampling in this interventional study. They were randomised into 2 groups one of which would receive the conventional protocol and the other group was to receive the experimental (modified) protocol for a period of 6 weeks, 3 times a week. Participants were chosen on the basis of inclusion and exclusion criteria.

\section{Tools and Equipment:}

The effects of the intervention on cervical range of motion were assessed using a goniometer. The effects on muscle endurance were done using cervical flexor test and cervical extensor endurance test. The Neck Disability Index (NDI) was adopted in this study to evaluate the level of disability. The change in level of pain was done using Visual Analog Scale (VAS). Proprioception was evaluated using a goniometer again. 

pain.

\section{Method:}

Approval was obtained from the Institutional Ethical Committee and informed written consent participants were recruited. A total of 40 participants were chosen on the basis of inclusion and the exclusion criteria. Participants were explained about the whole procedure and the demographic data which includes name, age, and gender was collected.

A pre-assessment protocol was followed and it included the following tools:

1. For pain: Visual analogue scale (VAS). After the pain scale was explained to them, the participants marked their current pain level by choosing a number from 0 (no pain at all) to 10 (unbearable pain), all 10 of which were displayed along a $10-\mathrm{cm}$ horizontal line. This scale is widely used in clinical settings, as it best reflects pain level and has been used as a valuable tool to assess effectiveness of pain treatment. ${ }^{[17,5]}$

2. For disability: The Neck Disability Questionnaire (NDI) is a modification of the Oswestry Low Back Pain Disability Index. It is a patient-completed, conditionspecific functional status questionnaire with 10 items including pain, personal care, lifting, reading, headaches, concentration, work, driving, sleeping and recreation. The NDI has sufficient support and usefulness to retain its current status as the most commonly used self-report measure for neck pain. ${ }^{[18]}$ Its levels of disability are classified as:

0-4points (0-8\%) no disability,

5-14points $(10-28 \%)$ mild

disability

15-24points (30-48\%) moderate

disability

25-34points (50- 64\%) severe

disability

35-50points (70-100\%) complete

disability
3. For Range of motion of cervical joints: Universal goniometer used for cervical ranges.

\section{For cervical flexor and extensor} endurance:

a] Deep cervical neck flexor endurance test: Patient was in supine, hook lying. For performing the test, tuck patients chin in and lift off table 1 inch. Patient was asked to hold the position for as long as he/she could without losing the chin tuck. The examiner looked for substitution of the platysma or SCM muscle. (Normal Values: Men: 38.9 seconds, Women: 29.4 seconds)

b] Cervical neck extensor endurance test: With the patient lying prone and head and neck past the edge of the table and the cervico-thoracic junction stabilized, the ability of the individual to sustain a chin tuck position in neutral for $20 \mathrm{~s}$ was evaluated. A positive finding for weakness of the deep neck extensors is the 'chin length' increasing with neck extension or simply the loss of the chin tuck, indicating a dominance of the superficial extensors of the neck. Weakness of both deep and superficial neck extensors was identified by the presence of neck flexion indicating an inability to hold the head up.

5. For proprioception: To measure the shoulder proprioception by a goniometer, the participant sat on a chair next to the therapeutic bed, with 1 arm on the bed at $45^{\circ}$ of abduction and $90^{\circ}$ of elbow flexion. The participant completed a total internal rotation to external rotation 3 times. The mean was recorded as the external rotation range of motion. Fifty percent of this range is considered the target angle. For keeping the angle in mind, the individual put their arm at this angle 3 times for 5 to 10 seconds with the guidance of the tester. the participant was asked to take the limb slowly to the desired angle. The 
mean error was recorded as absolute repositioning error.

6. Following the assessment, the participants were divided into 2 groups by randomized selection. Group $1 \quad(n=20)$ received only scapular stabilization exercises for neck pain for a span of 6 weeks, three times a week for half an hour per session. Group $2(n=20)$ received abdominal control feedback exercises along with scapula stabilization exercises for neck pain for a span of 6 weeks, three times per week for half an hour per session. Here the abdominal indrawing manoeuvre was always used along with exercises.

\section{For Conventional Protocol (Scapular Stabilization):}

1. Chin tucks: Move your chin towards your chest, holding for 5 seconds as you feel a comfortable stretch from your neck to the base of your skull.

2. Horizontal pull apart: By externally rotating the shoulders and supinating the forearms, pull away from the midline in an outward.

3. Serratus anterior punches: Stand like you're about to punch someone. Pivot to the right, and extend your left hand directly in front of you. Try to extend your arm as far out as it will go. Then pivot back to your left, bringing your left hand toward your chest and extending your right hand in front of you.

4. Retraction plus external rotation: Keeping the arms adducted, and keeping the forearms in midline initially, pull away towards the outside from the elbow while moving from pronation to supination.

5. TYI Exercises: Fully extend your elbows and fingers, positioning your palms facing inwards depress and retract your scapulae and attempt to hold these positions throughout the exercise. Align your head with your thoracic spine.
Mimic the shapes of T, Y and I with the arms and follow the procedure.

6. Chest press: Hold a pair of dumbbells above your chest with your arms straight. Your palms should face your feet and the weights should be just outside your shoulders. Lower the dumbbells to your chest, pause, and then press them back up to the starting position

7. Scapular retraction: Squeeze the shoulder blades together towards the midline as if trying to hold a coin between your shoulder blades. Hold for 5 seconds. Repeat 10 times.

For Modified Protocol (Scapular Stabilization along with Abdominal Control Feedback):

1. Wall presses: Inhale and, in one movement, press your body toward the wall as if doing a push-up. Exhale and push back to the original position, keeping your elbows close to your sides throughout

2. Knee push-ups: Hands and knees with eyes facing the floor. Place hands slightly greater than shoulder-width apart and knees comfortably apart. Slowly bend elbows and lower chest until chin reaches the ground, then slowly return to the starting position

3. Wall slides: Stand upright with back against a wall and feet shoulder-width apart. Bring arms up, pressing shoulder blades into the wall. The line of your upper arm, from your elbow to shoulder, should be perpendicular to the floor. Slowly bend knees and slide your back down the wall until your knees are bent at a 45-degree angle. As you bend your knees, straighten your elbows until your arms are extended straight up over your head, but still against the wall. Hold this position for 5 seconds. Straighten your knees to slide back up the wall until you are fully upright with knees straight and elbows bent back to their starting position. 
Sneha Ganu et.al. Effects of abdominal control feedback and scapular stabilization exercise on chronic neck pain.

*all the above given exercises are performed along with scapular stabilization protocol

Following the 6 weeks, a post assessment using the same tools was recorded.

\section{STATISTICAL METHODS}

The data obtained was entered, compared, coded, tabulated and analysed using Statistical Package for the Social Sciences (SPSS). Normality of the data was analysed using the Shapiro-Wilk test. Once the normality was drawn, for data that was normally distributed data within a group, paired $t$ test was used. For data that was normally distributed across groups, unpaired $t$ test was used. Wilcoxon test was used for distribution free intra-group data. Whereas for inter-group data that was distributed freely, Mann-Whitney U test was used.

\section{RESULT}

Represented below are the data findings of the Conventional group, Experimental group and Inter group comparison.

Table 1: Pre and Post values of Conventional Group

\begin{tabular}{|l|l|l|l|}
\hline Outcome Measures & Mean \pm Standard Deviation (PRE) & Mean \pm Standard Deviation (POST) & P value \\
\hline VAS & $5.3 \pm 0.865$ & $3.7 \pm 0.733$ & 0.000 \\
\hline ROM Flexion & $40.05 \pm 3.203$ & $42.5 \pm 2.502$ & 0.001 \\
\hline ROM Extension & $41.35 \pm 1.899$ & $43.55 \pm 1.669$ & 0.000 \\
\hline ROM Lateral Flexion (Right) & $39.05 \pm 2.964$ & $41.65 \pm 2.231$ & 0.000 \\
\hline ROM Lateral Flexion (Left) & $40.4 \pm 2.703$ & $42.7 \pm 1.525$ & 0.000 \\
\hline ROM Rotations (Right) & $61.75 \pm 4.482$ & $66.45 \pm 2.350$ & 0.000 \\
\hline ROM Rotations (Left) & $62.55 \pm 4.123$ & $67.8 \pm 2.016$ & 0.000 \\
\hline Endurance Time (DCFT) & $10.65 \pm 1.695$ & $17.1 \pm 1.411$ & 0.000 \\
\hline Endurance Time (CET) & $22.4 \pm 2.854$ & $30 \pm 1.806$ & 0.000 \\
\hline Proprioception & $5.8 \pm 1.576$ & $2.9 \pm 0.852$ & 0.000 \\
\hline NDI & $12.3 \pm 1.922$ & $7.05 \pm 1.050$ & 0.000 \\
\hline
\end{tabular}

Abbreviations: VAS: Visual Analog Scale; ROM: Range of motion; DCFT: Deep cervical flexor time; CET: Cervical extensor time; NDI: Neck Disability Index.

Inference: All the outcome measures show a significant improvement ( $p$ value $\leq 0.05$ ) when the conventional protocol is administered.

Table 2: Pre and Post values of Experimental Group

\begin{tabular}{|l|l|l|l|}
\multicolumn{2}{l}{ Table 2: Pre and Post values of Experimental Group } \\
\hline Outcome Measures & Mean \pm Standard Deviation (PRE) & Mean \pm Standard Deviation (POST) & P value \\
\hline VAS & $4.85 \pm 1.089$ & $2.05 \pm 0.759$ & 0.000 \\
\hline ROM Flexion & $39.5 \pm 2.351$ & $43.4 \pm 2.113$ & 0.001 \\
\hline ROM Extension & $41.95 \pm 2.724$ & $43.5 \pm 1.792$ & 0.001 \\
\hline ROM Lateral Flexion (Right) & $39.9 \pm 3.177$ & $43.05 \pm 1.572$ & 0.000 \\
\hline ROM Lateral Flexion (Left) & $40.65 \pm 2.519$ & $42.9 \pm 1.651$ & 0.000 \\
\hline ROM Rotations (Right) & $65.25 \pm 4.745$ & $69.8 \pm 2.608$ & 0.000 \\
\hline ROM Rotations (Left) & $67.15 \pm 3.869$ & $71.45 \pm 2.114$ & 0.000 \\
\hline Endurance Time (DCFT) & $10.9 \pm 2.553$ & $22.9 \pm 2.245$ & 0.000 \\
\hline Endurance Time (CET) & $23.85 \pm 3.199$ & $36.25 \pm 1.860$ & 0.000 \\
\hline Proprioception & $6.3 \pm 1.593$ & $3.05 \pm 0.826$ & 0.000 \\
\hline NDI & $10.65 \pm 2.539$ & $4.55 \pm 1.317$ & 0.000 \\
\hline
\end{tabular}

Abbreviations: VAS: Visual Analog Scale; ROM: Range of motion; DCFT: Deep cervical flexor time; CET: Cervical extensor time; NDI: Neck Disability Index.

Inference: All the outcome measures show a significant improvement ( $p$ value $\leq 0.05)$ when the experimental (modified) protocol is administered.

Table 3: Comparison between values of Mean Differences of Conventional Group and Experimental Group

\begin{tabular}{|l|l|l|l|}
\hline Outcome measures & Mean \pm Standard Deviation (Conventional) & Mean \pm Standard Deviation (Experimental) & P value \\
\hline VAS & $1.6 \pm 0.754$ & $2.8 \pm 1.281$ & 0.001 \\
\hline ROM Flexion & $2.45 \pm 1.849$ & $3.9 \pm 1.483$ & 0.113 \\
\hline ROM Extension & $2.2 \pm 1.576$ & $1.55 \pm 1.605$ & 0.185 \\
\hline ROM Lateral Flexion (Right) & $2.6 \pm 1.603$ & $3.15 \pm 2.368$ & 0.584 \\
\hline ROM Lateral Flexion (Left) & $2.3 \pm 1.780$ & $2.25 \pm 1.650$ & 0.989 \\
\hline ROM Rotations (Right) & $4.7 \pm 3.466$ & $4.55 \pm 2.724$ & 0.935 \\
\hline ROM Rotations (Left) & $5.25 \pm 3.109$ & $4.3 \pm 2.319$ & 0.353 \\
\hline Endurance Time (DCFT) & $6.45 \pm 1.572$ & $12 \pm 3.434$ & 0.000 \\
\hline Endurance Time (CET) & $7.6 \pm 2.703$ & $12.4 \pm 3.604$ & 0.000 \\
\hline Proprioception & $2.9 \pm 1.619$ & $3.25 \pm 1.482$ & 0.481 \\
\hline NDI & $5.25 \pm 2.149$ & $6.1 \pm 2.404$ & 0.199 \\
\hline
\end{tabular}



pain.

Abbreviations: VAS: Visual Analog Scale; ROM: Range of motion; DCFT: Deep cervical flexor time; CET: Cervical extensor time; NDI: Neck Disability Index.

Inference: VAS, Endurance time (DCFT) and Endurance time (CET) show a significant improvement ( $p$ value $\leq 0.05$ ) across the two groups i.e. experimental (modified) protocol is more effective as compared to conventional protocol with respect to the stated outcome measures.

\section{DISCUSSION}

The main objective of this study was to assess the effects of addition of abdominal control feedback (ACF) to the conventional scapular stabilization exercise protocol (SSE). Abdominal control feedback refers to a voluntary abdominal indrawing performed by the individual. It is achieved by recruiting the transversus abdominis muscle that belongs to the deep abdominal muscle layer. As a result, there was a marked improvement as far as pain and cervical flexor and extensor endurance was concerned when the abdominal control feedback protocol was added to the conventional protocol. Although the effects of the modified protocol were found to be superior, the scapular stabilization protocol was also proved to be of great effect when it came to the treatment of chronic mechanical neck pain. It not only helped in the improvement of pain and endurance of the neck musculature, but also with the range of motion of the cervical spine, level of disability and proprioception.

As far as the minimization of pain on movement of the cervical spine in a patient with chronic mechanical neck pain is concerned, the SSE is an effective method but the addition of ACF has proved to be more beneficial ( $p$ value $=0.002$ ). Pain reduction through exercise has been proved by research that explains the mechanism behind it. ${ }^{[19]}$ It says that the intrafusal fibers reset with exercise, thus breaking the cycle of muscle tension. Myogenic pain, according to Murphy BA in the year 2010, is associated with impaired circulation and accumulation of metabolites which is also taken care of by exercise. ${ }^{[20]}$ According to the study by Shirin Shiravi, the SA activation is superior when ACF is advocated. ${ }^{[21]}$ Serratus anterior (SA) is of essential importance when it comes to the scapular positioning and appropriate biomechanics. Since the samples presented with purely mechanical pain that resulted from faulty biomechanics at the cervical and shoulder joint, the activation of SA assisted with the correction of the scapular position thus helping with a greater reduction in associated pain symptoms than as compared to the conventional protocol.

The endurance time for both deep cervical flexors and extensors showed improvement in both the experimental groups, although the modified protocol was recorded to be of greater benefit ( $\mathrm{p}$ value for $\mathrm{DCFT}=0.000 ; \mathrm{p}$ value for $\mathrm{CET}=0.000$ ). According to Borrisut S (2013), improved endurance in both the experimental groups could be a result of improved coordination, increased motor unit recruitment and increased firing rate in each unit. This might have contributed to the increased endurance time by decreasing the muscle fatigue in chronic mechanical neck pain. ${ }^{[22-23]}$

The cervical range of motion: flexion, extension, lateral flexion, rotations; showed improvement in both the experimental groups. Although the addition of ACF did not show any significant change in the results. Rodriquez AA(1992), found that exercise helped in improving the endurance of neck muscles and thus in abatement of symptoms. ${ }^{[7]}$ There is an increase in the $\mathrm{Na}^{+}-\mathrm{K}^{+}$pump concentration and increase in the number of capillaries in the trapezius muscle. ${ }^{[24]}$ This also assist with quicker alleviation of metabolites and thus a reduced rate of fatigue and perception of pain thus helping in increased range.

Similarly, the discrepancy of the range for calculation of proprioception also shows a decline, thus implying that the proprioception shows improvement in both 

pain.

the experimental groups. There is no intergroup significance across the two experimental groups. Saleh MS also confirmed the improvement of proprioception when there was adequate exercise given to the deep cervical flexors. ${ }^{[25}$

The neck disability index was also used as an outcome measure and showed intragroup significance ( $\mathrm{p}$ value $=0.001$ in both the experimental groups) although no significance was noted across the groups.

\section{CONCLUSION}

Abdominal control feedback when added to scapular stabilization exercises show superior results in case of pain reduction and endurance improvement. It also restores efficient functioning and biomechanical patterns to the neck and shoulder joint.

\section{ACKNOWLEDGEMENT}

We would like to thank all the participants for their cooperation.

\section{Conflict of Interest: None}

\section{Source of Funding: None}

\section{Ethical Approval: Approved}

\section{REFERENCES}

1. Hoy DG, Protani M, De R, Buchbinder R. The epidemiology of neck pain. Best Pract Res Clin Rheumatol. 2010 Dec;24(6):78392

2. Cohen SP, Hooten WM. Advances in the diagnosis and management of neck pain. BMJ. 2017;358: j3221.

3. Bogduk, N. (2011). The Anatomy and Pathophysiology of Neck Pain. Physical Medicine and Rehabilitation Clinics of North America, 22(3):367-382.

4. Cohen, S. P. (2015). Epidemiology, Diagnosis, and Treatment of Neck Pain. Mayo Clinic Proceedings, 90(2):284-299.

5. Fathollahnejad, K., Letafatkar, A., \& Hadadnezhad, M. (2019). The effect of manual therapy and stabilizing exercises on forward head and rounded shoulder postures: a six-week intervention with a one-month follow-up study. BMC Musculoskeletal Disorders, 20(1):86.

6. Lin $\mathrm{IH}$, Chang $\mathrm{KH}$, Liou $\mathrm{TH}$, Tsou CM, Huang YC. The effect of progressive shoulder-neck exercise on cervical muscle functions of middle-aged and senior patients with chronic neck pain: a randomized controlled trial. Eur J Phys Rehabil Med. 2017;54(1):13-21.

7. Borna L, Ahmadi A, Sarafzadeh J, Maarufi $\mathrm{N}$. Comparison of cervical flexion relaxation phenomenon between forward head posture and healthy subjects [in Persian]. J Rehabil Med. 2016; 9:65-71.

8. Macdermid JC, Walton DM, Avery S, Blanchard A, Etruw E, McAlpine C, Goldsmith $\mathrm{CH}$. Measurement properties of the neck disability index a systematic review Journal of Orthopedic and Sports Physical Therapy. 2009 May;39(5):400-17.

9. Meyer J, Berk R, Anderson A. Recruitment patterns in the cervical paraspinal muscles during cervical forward flexion: evidence of cervical flexion-relaxation. Electromyogr Clin Neurophysiol. 1993; 33:217-223.

10. Murphy BA, Marshall PW, Taylor HH. The cervical flexion-relaxation ratio: reproducibility and comparison between chronic neck pain patients and controls. Spine. 2010; 35:2103-2108.

11. Mahmoud NF, Hassan KA, Abdelmajeed SF, Moustafa IM, Silva AG. The relationship between forward head posture and neck pain: A systematic review and meta-analysis. Curr Rev Musculoskelet Med. 2019;12(4):562-77.

12. Quek J, Pua Y-H, Clark RA, Bryant AL. Effects of thoracic kyphosis and forward head posture on cervical range of motion in older adults. Man Ther. 2013;18(1):65-71 J.

13. De-la-Llave-Rincón AI, Fernández-de-lasPeñas C, PalaciosCeña D, Cleland JA. Increased forward head posture and restricted cervical range of motion in patients with carpal tunnel syndrome. J Orthop Sport Phys Ther. 2009;39(9):658-64

14. Sheard, B., Elliott, J., Cagnie, B., \& O'Leary, S. (2012). Evaluating Serratus Anterior Muscle Function in Neck Pain Using Muscle Functional Magnetic Resonance Imaging. Journal of Manipulative and Physiological Therapeutics, 35(8):629-635. 
15. Struyf F. Scapular-focused treatment in patients with shoulder impingement syndrome: a randomized clinical trial. Clin Rheumatol. 2013; 32:73-85.

16. Vega Toro AS. Instruction and feedback for conscious contraction of the abdominal muscles increases the scapular muscles activation during shoulder exercises. Man Ther. 2016:11-18.

17. Bijur PE, Silver W, Gallagher EJ. Reliability of the visual analog scale for measurement of acute pain. Academic emergency medicine. $2001 \mathrm{Dec} ; 8(12): 1153$ 7.

18. Vernon H, Mior S. The Neck Disability Index: a study of reliability and validity. Journal of manipulative and physiological therapeutics. 1991 Sep:409-15.

19. Borisut S, Vongsirinavarat M, Vachalathiti R, Sakulsriprasert P. Effects of strength and endurance training of superficial and deep neck muscles on muscle activities and pain levels of females with chronic neck pain. Journal of physical therapy science. 2013; 25(9):1157-62.

20. McCarthy JP, Pozniak MA, Agre JC, et al.: Neuromuscular adaptation to concurrent strength and endurance training. Med Sci Sports Exerc, 2002, 34:511-519.

21. Shiravi S, Letafatkar A, Bertozzi L, Pillastrini P, Khaleghi Tazji M. Efficacy of
Abdominal Control Feedback and Scapula Stabilization Exercises in Participants with Forward Head, Round Shoulder Postures and Neck Movement Impairment. Sports health. 2019 May;11(3):272-9.

22. Kadi F, Ahlgren $\mathrm{C}$, Waling $\mathrm{K}$, et al.: The effects of different training programs on the trapezius muscle of women with workrelated neck and shoulder myalgia. Acta Neuropathol, 2000, 100: 253-258.

23. Rodriquez AA, Bilkey WJ, Agre JC. Therapeutic exercise in chronic neck and back pain. Archives of physical medicine and rehabilitation. 1992 Sep 1;73(9):870-5.

24. Ylinen J. Physical exercises and functional rehabilitation for the management of chronic neck pain. Europa medicophysica. 2007 Mar 1;43(1):119-32.

25. Saleh MS, Rehab NI, Sharaf MA. Effect of deep cervical flexors training on neck proprioception, pain, muscle strength and dizziness in patients with cervical spondylosis: A randomized controlled trial. Physical Therapy and Rehabilitation 5(1):14. 2018 Jan:5-14.

How to cite this article: Ganu S, Gor U. Effects of abdominal control feedback and scapular stabilization exercise on chronic neck pain. Int $J$ Health Sci Res. 2021; 11(6): 318-325. DOI: https://doi.org/10.52403/ijhsr.20210647 